\title{
A Node Importance Measuring Method based on Hypernetwork
}

\author{
Quan Xiao, Fangli Yang, Song Luo, Lihong Zhang, Hua Zhao and Wei Shu \\ School of Information Technology, Jiangxi University of Finance and Economics, \\ 330032 Nanchang, China \\ xiaoquan@foxmail.com
}

\begin{abstract}
Measuring the importance of nodes in network is an important issue in the study of complex networks. The networks in current researches are mostly based on classical graph theory, which have limitations on describing certain complex relations. In this work, we introduce hypernetwork, taking hypergraph as representation foundation, to describe relations. Hypernetwork is able to extend the modeling and describing capability of traditional network and may be a promising representation model of complex relations. However, a general lack of attention to the node importance measuring in hypernetwork, an important fundamental issue for its further application, has been noted across the majority of related published works. In this paper, we utilize the idea of deleting method, to measure the importance of node in hypernetwork through investigating the influence on the whole network when deleting it. Specifically, the influence is measured by direct loss and indirect loss. Through a calculating example, our method is compared with node degree, betweenness, closeness centrality, degree of neighbor nodes etc., the result shows this method has better adaptability and accuracy.
\end{abstract}

Keywords: hypernetwork; node importance measuring; node deleting

\section{Introduction}

Today, people are living in a world full of all kinds of networks: from the Internet to WWW, from large-scale power networks to the global transportation network, from organisms in brain to metabolic network, from research collaboration network, to various economic, political and social networks-- complex networks are everywhere. Research on network has become a common concern and research hotspot of many subjects such as physics, computer science, biology, chemistry, economics and sociology. In network research, evaluating the importance of network nodes and mining the important ones is a basic issue. Node importance measuring is an good way to investigate network reliability and robustness, meanwhile, to identify, protect and maintain important nodes is of great significance to improve the flow of information and reduce information exchange cost.

With the increase of network complexity, the constructed networks specific to different application background are becoming progressively more complex over time [1], the traditional graph theory based network, in which an edge connects a pair of nodes, has gradually shown its modeling limitations. Taking scientific collaboration network as an example, it is easy to represent whether two authors have co-author relationships by two nodes and a line connects them. But the traditional network is difficult to describe the situation that more than two authors write one paper collaboratively. The proposition of hypergraph and hypernetwork, in which a hyperedge can contain more than two nodes, makes it possible to study the complex relationships. Thus, the collaboration relationships can be represented as a hypernetwork in which nodes represent authors and hyperedges represent papers that have been coauthored by the groups of authors [2]. Hypernetwork can be defined as "network beyond network" or "network of network" [3-4], and it has characteristics of nest, multi-level, multi-layer and multi-attribute that classical graph and 
network do not possess. For the present, supply chain hypernetwork, financial hypernetwork, power supply hypernetwork, population migration hypernetwork, interpersonal hypernetwork, and knowledge hypernetwork has been proposed and studied by many researchers [5].

For kinds of hypernetworks, the node importance measuring is also an important fundamental issue to be investigated. For example, who takes the authoritative position in a research field in scientific collaboration hypernetwork? How to identify and protect the hidden danger points in hypernetwork security? Although there emerge some methods in the traditional network, such as deleting methods, SNA based methods, and indicators such as degree, betweenness, clustering coefficient, unconnected node pairs, spanning trees reduce value etc., there is still a lack of attention on the node importance measuring in hypernetwork. The objective of this study is to investigate the method for measuring node importance in hypernetwork model, which may help answering the questions above, and furthermore make some contributions to hypernetwork theories and applications.

Based on hypernetwork model, in this paper we proceed our study from the perspective of overall network. In order to distinguish from single measuring indicators, we introduce direct loss and indirect loss when deleting a node to achieve a comprehensive weighted measuring. Our method takes the influence of node to the overall network into consideration, and can effectively measure the importance of node itself.

The remainder of our paper is organized as follows. In Section 2 the related works are reviewed. The detailed measuring method is demonstrated In Section 3. In Section 4 we give a calculation instance and discuss the results. Finally the conclusions are drawn in Section 5.

\section{Related Researches}

\subsection{Node Importance Measuring in Network}

Theoretically, node importance measuring methods can be classified into two categories. The first category contains social network analysis (SNA) based methods. In these methods, the integrality and connectivity of the network will not be destroyed and the importance of node is equivalent to its significance in the network [6]. Such as Jun et al. [7] proposed a unified multiple metrics for evaluating framework of node importance with non-conflict equivalent classes. To measure the contextual partner importance in scientific collaboration networks, Schall [8] present a context-based metric to measure the importance of partners, in which an established graph models such as the notion of hubs and authorities provide the basis for the measurement. Agryzkov [9] proposed a centrality index called ranking-betweenness centrality, combining random-walk betweenness centrality and adapted PageRank algorithm together, for urban street patterns represented as networks in geographical space.

The second category of theoretical research can be called deleting based methods. The importance of a node is derived from the influence to the network when deleting it, i.e. importance is equivalent to destructiveness. Li et al. [10] considered that to destroy node with different distance would bring different losses to network, and took the inverse of distance between node pair as weight value, and then calculated the weighted sum of all disconnected node pairs to measure the destructiveness to the network connectivity. Qi [11] propose a centrality measurement called spanning tree centrality (STC) for weighted networks. The STC score of a node in a network is defined as the number of spanning trees with the node $\mathrm{v}$ as a cut node. Saito [12] propose an method named "super-mediators" to discover influential nodes in a social network. The "super-mediators" are defined as those nodes that if removed will greatly decrease information spread. The measurement is a problem of difference maximization of the average influence degree with respect to removal of a node. 
In general, the classical indicators for measuring node importance in network include degree, betweenness, centrality, subgraph, average shortest distance etc., and some related indicators proposed for specific problems. Each indicator mainly focuses on some certain characteristics of the network, such as the position in network structure, the influence of network in information diffusion, or the contribution in subnetwork. As different measuring methods may have different results and sometimes the difference is quite notable [13], in many researches the importance of nodes are measured through the combination of multiple indicators according to the network characteristics. For hypernetwork, there is still a general lack of attention on the node importance measuring issue.

\subsection{Hypernetwork}

With deeper investigation into network theories and applications, the constructed networks specific to different fields are becoming increasingly complex [1] - there are more nodes of different types in network, and the connections between them are diversified. There exist complex behaviors such as clustering, nesting and reduction for nodes and edges. In these cases, relations between nodes are not just binary, but may be multi-element, multi-layer or multi-granularity, which cannot be properly coped by traditional networks. The emergence of hypergraph and hypernetwork facilitate investigation in problems with these properties. The concept of hypergraph was firstly proposed by Berge [14], and Sheffi define hypernetwork as "a network beyond existing network" and "network in network" [3-4]. A network represented by hypergraph can be called as hypernetwork. Hypergraph and hypernetwork take the advantage of representing complex characteristics beyond the capability of traditional graph and network [15].

Taking scientific research collaboration as an example, as a hyperedge in hypernetwork can contain more than two nodes, so it is useful to represent the collaboration network as a hypernetwork in which nodes represent authors and hyperedges represent papers that have been coauthored by the groups of authors [2].

In traditional network, if a paper is co-authored by 100 authors, the node degree of each of the 100 authors is at least 99. This high degree value cannot reflect the objective influence of them. Whereas in hypernetwork, we can draw only one hyperedge including the 100 nodes to represent the co-authored paper. Although there exists collaboration by many people, the hyperedge degree will be only 1 , thus the real influence of authors can be reflected. In this scientific research collaboration hypernetwork, nodes represent authors, while hyperedges can not only represent the co-authored paper, but can clearly depict the collaborative relations between authors, while in traditional network, co-authored paper and collavorative relations are confused. Bipartite graph are proposed to solve the confusion, but it will break the homogeneity of nodes, which will result in ineffectiveness of some parameters and generation of ambiguity when calculating connectivity, centrality and other topological properties [16]. As a modeling tool beyond traditional network, hypernetwork can represent interactions and influences between different networks, so far hypernetwork has been applied to supply-chain management, financial analysis, power supply designing, population migration research, knowledge management etc., and many domain specific hypernetworks has been constructed [5].

Because of different properties between traditional network and hypernetwork, the importance measuring indicators in traditional network cannot be directly transplanted to hypernetwork. However, it is undoubtedly an essential fundamental matter for deeper application of hypernetwork to measure the importance of internal nodes, yet there is still a lack of attention on this issue. In this paper we are trying to make some exploration about this ignored but important question. 


\section{Node Importance Measuring Method}

\subsection{Basic Idea}

According to the deleting based methods, the importance of a node is the destructiveness to the network when deleting it. For a network, when a node in it is deleted, it will cause three aspects of damages to the whole network connectivity. Firstly, when a node deleted, there will be no relationship between it and other nodes, which is the most direct loss to be observed. Secondly, some nodes were originally connected, but when deleting a node, some of the rest nodes may be unconnected because of the loss of the role as bridge of the deleting one. Thirdly, the deleting of a node may cause the increasing of distances between other nodes. The latter two cases are relatively indirect to observe [17-18]. We can call the loss in the first situation as Direct Loss (DL), and the latter two kinds of loss as Indirect Loss (IL). DL and TL are integrated as Total Loss (TL), which represents total destructiveness to the whole network connectivity [19]. Through the extent of damage, we can measure the importance of the node.

\subsection{Definition of Fundamental Concepts}

According to the concept of hypernetwork proposed by Sheffi [3], we give the definition of hypernetwork as: A hypernetwork $\mathrm{HN}=(\mathrm{V}, \mathrm{E})$ on a set $\mathrm{V}$ is a family $\mathrm{e}_{\mathrm{j}}$ called hyperedge of non- empty subsets of $V$, where $V=\left\{V_{1}, V_{2}, \ldots, V_{n}\right\}$ is a finite set of nodes, $E=\left\{e_{1}, e_{2}, \ldots, e_{m}\right\}$ is a family of subset of $V$, s.t. $e_{j} \neq \emptyset, j=1,2, \ldots, m$, and $\mathrm{U}_{\mathrm{j}} \mathrm{e}_{\mathrm{j}}=\mathrm{V}$.

In Figure 1 it is a hypernetwork composed of 6 points and 9 hyperedges, where $\mathrm{V}=\left\{v_{1}, v_{2}, \ldots, v_{6}\right\}$ and $\mathrm{E}=\left\{e_{1}, e_{2}, \ldots, e_{9}\right\}=\left\{\left\{v_{1}, v_{2}\right\},\left\{v_{2}, v_{4}\right\}, \ldots,\left\{v_{4}, v_{5}, v_{6}\right\}\right\}$.

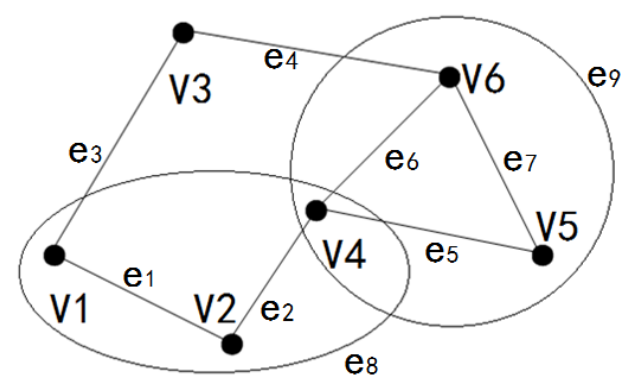

\section{Figure 1. An Example of Hypernetwork}

Besides the forms of set and diagram, for scientific or engineering calculation tasks, hypernetwork is often handled as matrix. Adjacent matrix $(A M)$, distance matrix $(D M)$ and incidence matrix $(I M)$ are useful for calculation of the node importance in our work, to facilitate the elaboration of measuring method, we give their basic definition as follows.

$A M$ is the basic matrix representation of graph, and is used to describe the relationship between nodes in the graph, The element $a m_{i j}$ in $A M$ is defined as:

$$
a m_{i j}=\left\{\begin{array}{ll}
n, & v_{i} \text { and } v_{j} \text { are adjacent for } n \text { times } \\
0, & v_{i} \text { and } v_{j} \text { are not adjacent }
\end{array} .\right.
$$

$A M$ describes the direct relationship between nodes in a network.

The $A M$ of the hypernetwork in Figure 1 is: 


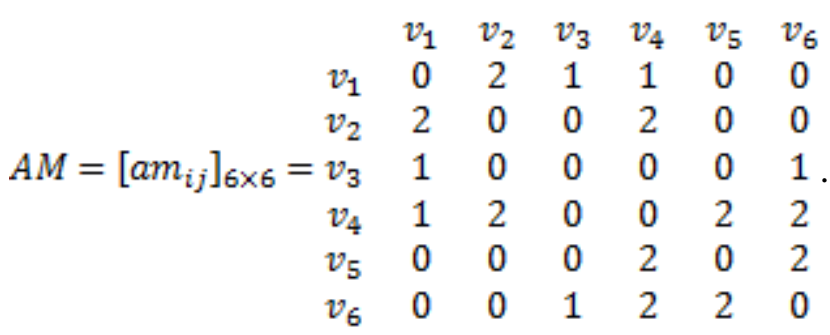

$D M$ is a matrix that describes the shortest distance between nodes in a connected network. Elements in $D M$ is defined as:

$$
d m_{i j}=\left\{\begin{array}{cc}
\operatorname{dist}(i, j), & i \neq j \\
0, & i=j
\end{array}\right.
$$

where $\operatorname{dist}(i, j)$ is the shortest distance between $v_{i}$ and $v_{j}$.

The $D M$ of the hypernetwork in Figure 1 is:

$$
\begin{aligned}
& \begin{array}{llllll}
v_{1} & v_{2} & v_{3} & v_{4} & v_{5} & v_{6}
\end{array} \\
& \begin{array}{lllllll}
v_{1} & 0 & 1 & 1 & 1 & 2 & 2
\end{array} \\
& \begin{array}{lllllll}
v_{2} & 1 & 0 & 2 & 1 & 2 & 2
\end{array}
\end{aligned}
$$

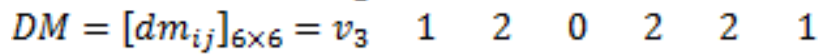

$$
\begin{aligned}
& \begin{array}{lllllll}
v_{4} & 1 & 1 & 2 & 0 & 1 & 1
\end{array} \\
& \begin{array}{lllllll}
v_{5} & 2 & 2 & 2 & 1 & 0 & 1
\end{array} \\
& \begin{array}{lllllll}
v_{6} & 2 & 2 & 1 & 1 & 1 & 0
\end{array}
\end{aligned}
$$

IM shows the composition relationship between hyperedges and nodes, in hypernetwork $H N=(V, E)$, the elements in $I M$ is defined as:

$$
i m_{i j}=\left\{\begin{array}{cc}
1, & v_{j} \text { is an element in hyperedge } e_{i} \\
0, & v_{j} \text { is not in } e_{i}
\end{array} .\right.
$$

The $I M$ of the hypernetwork in Figure 1 is:

$$
I M=\left[i m_{i j}\right]_{9 \times 6}=\begin{array}{ccccccc} 
& v_{1} & v_{2} & v_{3} & v_{4} & v_{5} & v_{6} \\
e_{1} & 1 & 1 & 0 & 0 & 0 & 0 \\
e_{2} & 0 & 1 & 0 & 1 & 0 & 0 \\
e_{3} & 1 & 0 & 1 & 0 & 0 & 0 \\
e_{4} & 0 & 0 & 1 & 0 & 0 & 1 \\
e_{5} & 0 & 0 & 0 & 1 & 1 & 0 \\
e_{6} & 0 & 0 & 0 & 1 & 0 & 1 \\
e_{7} & 0 & 0 & 0 & 0 & 1 & 1 \\
e_{8} & 1 & 1 & 0 & 1 & 0 & 0 \\
e_{9} & 0 & 0 & 0 & 1 & 1 & 1
\end{array} .
$$

\subsection{Measuring algorithm Description}

For hypernetwork $\mathrm{HN}=(\mathrm{V}, \mathrm{E})$, when deleting node $v_{i}$, the generated subnetwork is $\mathrm{HN}^{\prime}=\left(\mathrm{V}^{\prime}{ }^{\prime} \mathrm{E}^{\prime}\right)$, where $\mathrm{V}^{\prime}=\mathrm{V}-v_{i}$ is the node set of $\mathrm{HN}^{\prime}$ and $\mathrm{E}^{\prime}$ is the hyperedge set of $\mathrm{HN}^{r}$. $\mathrm{E}^{\prime}$ is composed of hyperedges in $\mathrm{E}$ subtracting that linked with $v_{i}$. For hyperedge with more than three nodes, removing one node will not affect the adjacent relation between the other two. The calculating procedure of our node importance measuring algorithm is as follows:

(1) Input the $I M$ of hypernetwork $\mathrm{HN}$, and then calculate $A M$ and $D M$.

(2) For deleting node $v_{i}$, sum up the reciprocal of all elements in row $i$, or column $i$ then get the $D L$ value of deleting $v_{i}$.

(3) In $I M$, deleting the column where $v_{i}$ located to obtain the incidence matrix $I M^{r}$ 
of $\mathrm{HN}^{\prime}$, and then calculate $A M^{r}$ and $D M^{\prime}$ of $\mathrm{HN}^{\prime}$.

(4) For the upper triangular part of $D M$ and $D M^{r}$, find all the corresponding elements $d m_{i j}$ in $D M$ and $d m_{i j}^{v}$ in $D M^{v}$ that satisfy $d m_{i j}^{v}>d m_{i j}$, to sum up $1 / d m_{i j}^{\prime}-1 / d m_{i j}$ of all found $d m_{i j}$ and $d m_{i j}^{\prime}$ as the IL value.

(5) Calculate total loss $T L=D L+I L$, which is the importance value of $v_{i}$.

The Python pseudo-code of the measuring algorithm is shown in Figure 2:

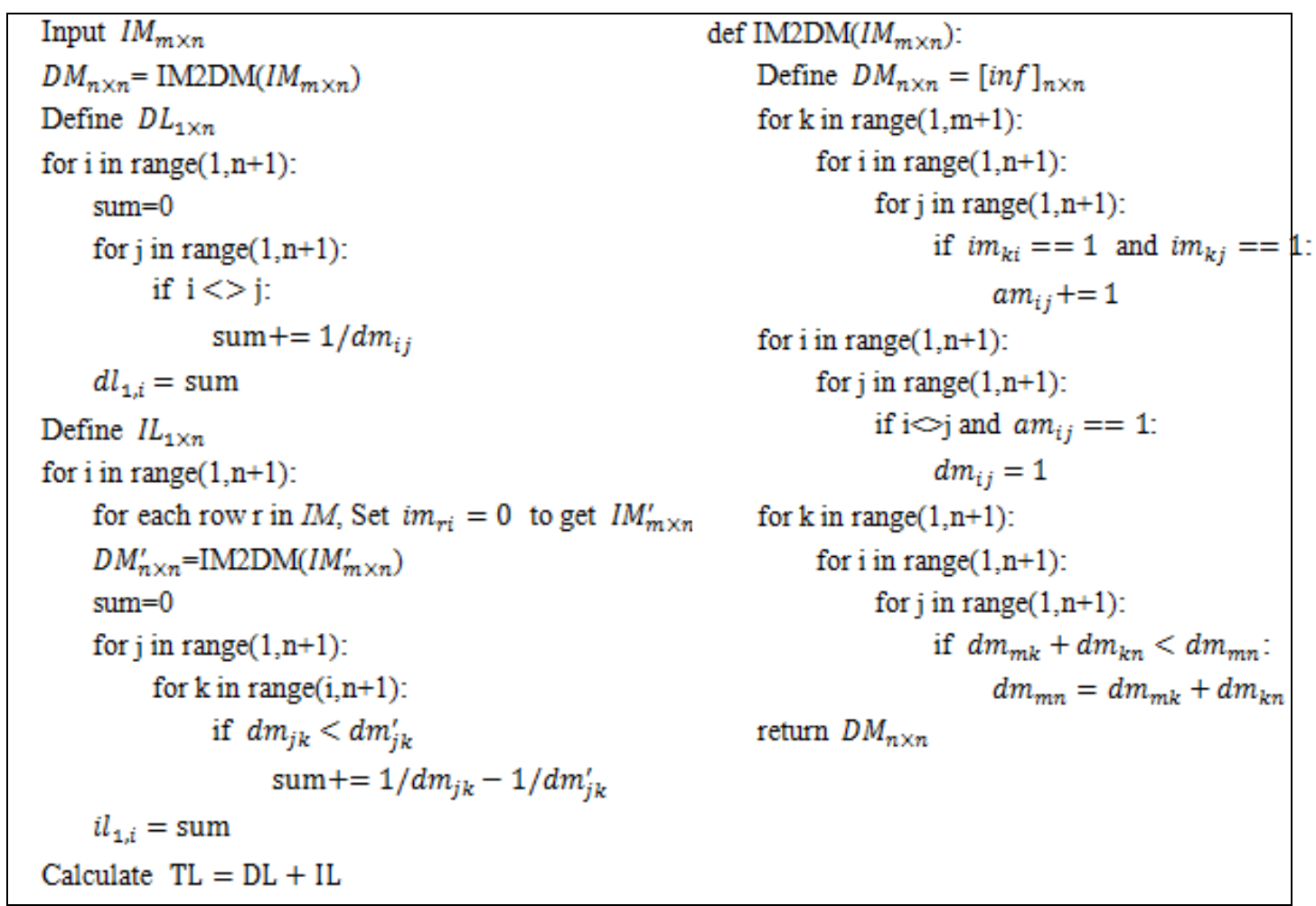

Figure 2. Python pseudo-code of the Measuring Algorithm

\subsection{Normalization of Total Loss}

The purpose of normalization is to facilitate comparing between networks with different sizes and eliminate the influence of network size to make the indicator valued from 0 to 1 . For a hypernetwork with $n$ nodes, the maximum total loss will result from deleting the core node from the star-network. In this situation, the total loss will be:

$$
T L_{\max }=(n-1)+\frac{(n-1)(n-2)}{2} \cdot \frac{1}{2}=\frac{(n-1)(n+2)}{4}
$$

So the normalized total loss is:

$$
T L^{\prime}=\frac{T L}{T L_{\max }}=\frac{4 \cdot T L}{(n-1)(n+2)}
$$

\section{Calculation Instance}

\subsection{Illustrated Hypernetwork}

We illustrate the calculation process and effectiveness of our method with an example of scientific research collaboration hypernetwork. We collect the co-author data of a research organization, and construct a scientific research collaboration hypernetwork as Figure 3 shows, where nodes $v_{1}, v_{2}, \ldots, v_{18}$ represent 18 different researchers and the 
hyperedges represent the co-authored relations between them. For example, researcher $v_{5}$ not only has direct collaboration with $v_{8}$, but also collaborates with $v_{2}, v_{3}, v_{7}, v_{10}$ and $v_{12}$. Researcher $v_{5}$ also plays an indirect role in the collaboration between $v_{7}$ and $v_{8}$. Therefore, when measuring node importance in the whole network, we should consider both direct and indirect the influence of the node on the whole hypernetwork.

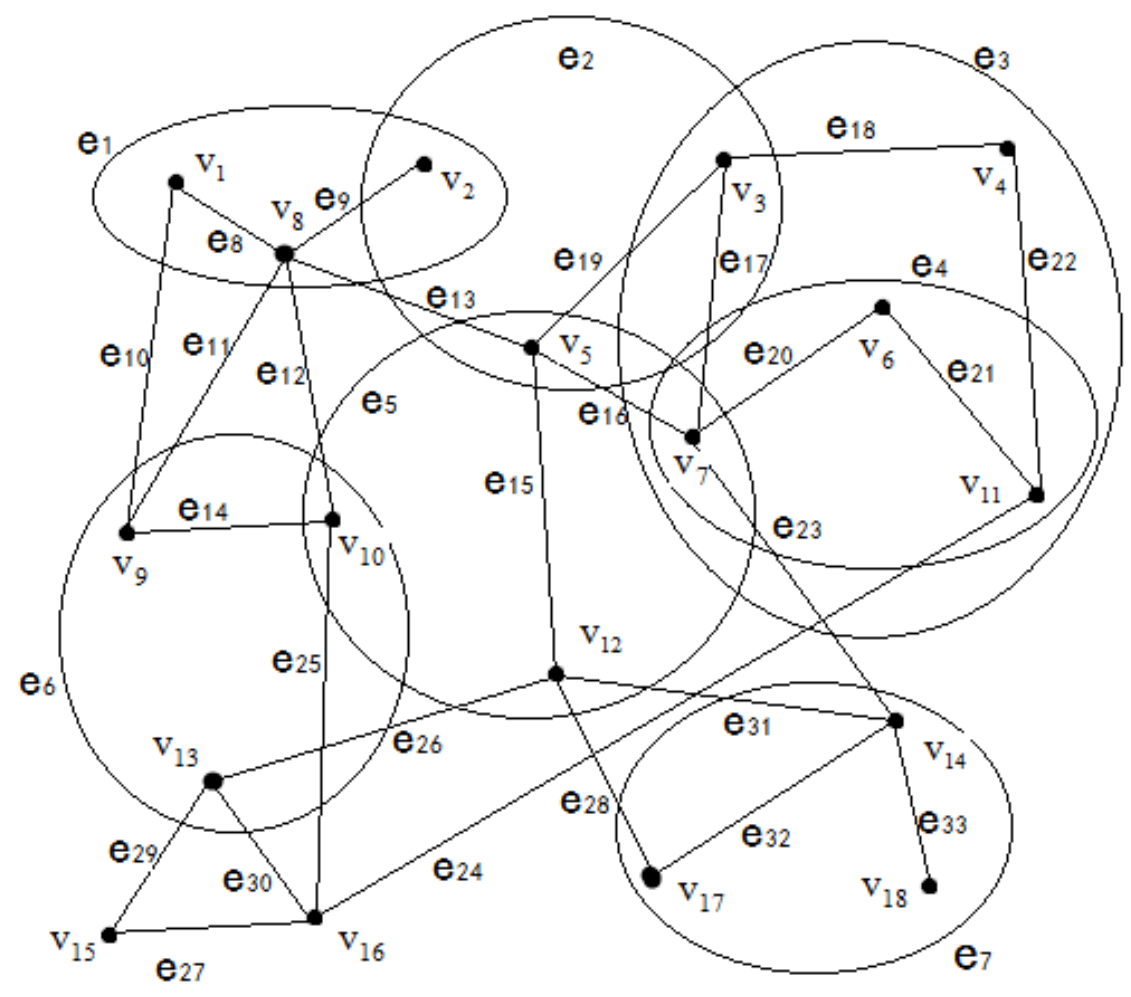

Figure 3. Scientific Research Collaboration Hypernetwork

\subsection{Calculation Results and Discussions}

According to the measuring method proposed above, the importance value of each node in hypernetwork in Figure 3 are calculated, which is compared with some commonly used node importance indicators as Table 1 shows.

Table 1. Result of Node Importance Measuring

\begin{tabular}{|c|c|c|c|c|c|c|c|c|c|c|c|c|c|}
\hline \multicolumn{2}{|c|}{ ND } & \multicolumn{2}{|c|}{ NND } & \multicolumn{2}{|c|}{$\mathrm{BC}$} & \multicolumn{2}{|c|}{$\mathrm{CC}$} & \multicolumn{2}{|c|}{ DL } & \multicolumn{2}{|c|}{ IL } & \multicolumn{2}{|c|}{ TL } \\
\hline $\begin{array}{l}\text { No } \\
\text { de }\end{array}$ & value & $\begin{array}{c}\text { Nod } \\
\mathrm{e}\end{array}$ & $\begin{array}{c}\text { valu } \\
\text { e }\end{array}$ & $\begin{array}{c}\text { Nod } \\
\mathrm{e}\end{array}$ & value & $\begin{array}{c}\text { Nod } \\
\mathrm{e}\end{array}$ & value & $\begin{array}{c}\text { Nod } \\
\mathrm{e}\end{array}$ & value & $\begin{array}{c}\text { Nod } \\
\mathrm{e}\end{array}$ & value & $\begin{array}{c}\text { Nod } \\
\mathrm{e}\end{array}$ & value \\
\hline v7 & 7 & v7 & 38 & v7 & $\begin{array}{c}0.642 \\
9\end{array}$ & v7 & $\begin{array}{c}0.548 \\
7\end{array}$ & v13 & $\begin{array}{c}11.833 \\
3\end{array}$ & v13 & $\begin{array}{c}4.250 \\
0\end{array}$ & v13 & $\begin{array}{c}0.189 \\
2\end{array}$ \\
\hline v5 & 6 & v10 & 32 & v10 & $\begin{array}{c}0.642 \\
9\end{array}$ & v13 & $\begin{array}{c}0.534 \\
8\end{array}$ & v7 & $\begin{array}{c}12.166 \\
7\end{array}$ & v7 & $\begin{array}{c}2.000 \\
0\end{array}$ & v7 & $\begin{array}{c}0.166 \\
7\end{array}$ \\
\hline v8 & 6 & v5 & 31 & v13 & $\begin{array}{c}0.642 \\
9\end{array}$ & v12 & $\begin{array}{c}0.465 \\
5\end{array}$ & v10 & $\begin{array}{c}11.833 \\
3\end{array}$ & v10 & $\begin{array}{c}0.333 \\
3 \\
\end{array}$ & v10 & $\begin{array}{c}0.143 \\
1\end{array}$ \\
\hline v3 & 5 & v12 & 30 & v5 & $\begin{array}{c}0.620 \\
7\end{array}$ & v10 & $\begin{array}{c}0.342 \\
0\end{array}$ & v12 & $\begin{array}{c}11.166 \\
7\end{array}$ & v12 & $\begin{array}{c}0.600 \\
0\end{array}$ & v12 & $\begin{array}{c}0.138 \\
4\end{array}$ \\
\hline $\mathrm{v} 10$ & 5 & v3 & 28 & $\mathrm{v} 12$ & 0.600 & v14 & 0.277 & v6 & 11.166 & v6 & 0.333 & v6 & 0.135 \\
\hline
\end{tabular}




\begin{tabular}{cc|cc|cc|cc|cc|cc|cc}
\hline & & & & 0 & 5 & 7 & & 3 & 3 \\
\hline $\mathrm{v} 11$ & 5 & $\mathrm{v} 11$ & 23 & $\mathrm{v} 6$ & $\begin{array}{c}0.600 \\
0\end{array}$ & $\mathrm{v} 6$ & $\begin{array}{c}0.257 \\
1\end{array}$ & $\mathrm{v} 5$ & $\begin{array}{c}11.333 \\
3\end{array}$ & $\mathrm{v} 5$ & 0 & $\mathrm{v} 5$ & $\begin{array}{c}0.133 \\
3\end{array}$ \\
\hline $\mathrm{v} 12$ & 5 & $\mathrm{v} 8$ & 21 & $\mathrm{v} 3$ & $\begin{array}{c}0.562 \\
5\end{array}$ & $\mathrm{v} 5$ & $\begin{array}{c}0.188 \\
0\end{array}$ & $\mathrm{v} 3$ & $\begin{array}{c}10.833 \\
3\end{array}$ & $\mathrm{v} 3$ & 0 & $\mathrm{v} 3$ & $\begin{array}{c}0.127 \\
5\end{array}$ \\
\hline $\mathrm{v} 14$ & 5 & $\mathrm{v} 4$ & 21 & $\mathrm{v} 11$ & $\begin{array}{c}0.514 \\
3\end{array}$ & $\mathrm{v} 8$ & $\begin{array}{c}0.151 \\
8\end{array}$ & $\mathrm{v} 11$ & $\begin{array}{c}10.000 \\
0\end{array}$ & $\mathrm{v} 11$ & $\begin{array}{c}0.333 \\
3\end{array}$ & $\mathrm{v} 11$ & $\begin{array}{c}0.121 \\
6\end{array}$ \\
\hline $\mathrm{v} 6$ & 4 & $\mathrm{v} 6$ & 20 & $\mathrm{v} 4$ & $\begin{array}{c}0.500 \\
0\end{array}$ & $\mathrm{v} 3$ & $\begin{array}{c}0.148 \\
3\end{array}$ & $\mathrm{v} 8$ & 9.9167 & $\mathrm{v} 8$ & $\begin{array}{c}0.333 \\
3\end{array}$ & $\mathrm{v} 8$ & $\begin{array}{c}0.120 \\
6\end{array}$ \\
\hline $\mathrm{v} 13$ & 4 & $\mathrm{v} 2$ & 20 & $\mathrm{v} 8$ & $\begin{array}{c}0.500 \\
0\end{array}$ & $\mathrm{v} 2$ & $\begin{array}{c}0.145 \\
9\end{array}$ & $\mathrm{v} 14$ & 9.4167 & $\mathrm{v} 14$ & $\begin{array}{c}0.500 \\
0\end{array}$ & $\mathrm{v} 14$ & $\begin{array}{c}0.116 \\
7\end{array}$ \\
\hline $\mathrm{v} 9$ & 4 & $\mathrm{v} 13$ & 20 & $\mathrm{v} 9$ & $\begin{array}{c}0.486 \\
5\end{array}$ & $\mathrm{v} 9$ & $\begin{array}{c}0.115 \\
0\end{array}$ & $\mathrm{v} 2$ & 9.3333 & $\mathrm{v} 2$ & $\begin{array}{c}0.416 \\
7\end{array}$ & $\mathrm{v} 2$ & $\begin{array}{c}0.114 \\
7\end{array}$ \\
\hline $\mathrm{v} 16$ & 4 & $\mathrm{v} 9$ & 18 & $\mathrm{v} 14$ & $\begin{array}{c}0.486 \\
5\end{array}$ & $\mathrm{v} 11$ & $\begin{array}{c}0.078 \\
3\end{array}$ & $\mathrm{v} 9$ & 9.4167 & $\mathrm{v} 9$ & $\begin{array}{c}0.166 \\
7\end{array}$ & $\mathrm{v} 9$ & $\begin{array}{c}0.112 \\
7\end{array}$ \\
\hline $\mathrm{v} 4$ & 3 & $\mathrm{v} 14$ & 17 & $\mathrm{v} 16$ & $\begin{array}{c}0.486 \\
5\end{array}$ & $\mathrm{v} 17$ & $\begin{array}{c}0.070 \\
8\end{array}$ & $\mathrm{v} 4$ & 9.5000 & $\mathrm{v} 4$ & 0 & $\mathrm{v} 4$ & $\begin{array}{c}0.111 \\
8\end{array}$ \\
\hline $\mathrm{v} 2$ & 3 & $\mathrm{v} 16$ & 16 & $\mathrm{v} 2$ & $\begin{array}{c}0.473 \\
7\end{array}$ & $\mathrm{v} 16$ & $\begin{array}{c}0.043 \\
3\end{array}$ & $\mathrm{v} 16$ & 9.0833 & $\mathrm{v} 16$ & 0 & $\mathrm{v} 16$ & $\begin{array}{c}0.106 \\
9\end{array}$ \\
\hline $\mathrm{v} 1$ & 3 & $\mathrm{v} 1$ & 13 & $\mathrm{v} 17$ & $\begin{array}{c}0.418 \\
6\end{array}$ & $\mathrm{v} 1$ & $\begin{array}{c}0.012 \\
9\end{array}$ & $\mathrm{v} 1$ & 8.2000 & $\mathrm{v} 1$ & 0 & $\mathrm{v} 1$ & $\begin{array}{c}0.096 \\
5\end{array}$ \\
\hline $\mathrm{v} 17$ & 3 & $\mathrm{v} 17$ & 12 & $\mathrm{v} 1$ & $\begin{array}{c}0.409 \\
1\end{array}$ & $\mathrm{v} 4$ & $\begin{array}{c}0.000 \\
0\end{array}$ & $\mathrm{v} 17$ & 8.1667 & $\mathrm{v} 17$ & 0 & $\mathrm{v} 17$ & $\begin{array}{c}0.096 \\
1\end{array}$ \\
\hline $\mathrm{v} 15$ & 2 & $\mathrm{v} 15$ & 8 & $\mathrm{v} 15$ & $\begin{array}{c}0.409 \\
1\end{array}$ & $\mathrm{v} 15$ & $\begin{array}{c}0.000 \\
0\end{array}$ & $\mathrm{v} 15$ & 7.2500 & $\mathrm{v} 15$ & 0 & $\mathrm{v} 15$ & $\begin{array}{c}0.085 \\
3\end{array}$ \\
\hline $\mathrm{v} 18$ & 2 & $\mathrm{v} 18$ & 8 & $\mathrm{v} 18$ & $\begin{array}{c}0.346 \\
2\end{array}$ & $\mathrm{v} 18$ & $\begin{array}{c}0.000 \\
0\end{array}$ & $\mathrm{v} 18$ & 6.7833 & $\mathrm{v} 18$ & 0 & $\mathrm{v} 18$ & $\begin{array}{c}0.079 \\
8\end{array}$ \\
\hline
\end{tabular}

Note: ND - node degree; NND - neighbor node degree; BC - betweenness centrality; CC - closeness centrality; DL - direct loss, IL - indirect loss, TL - normalized total loss.

From the calculation results we can see that there exist some differences of the importance value under different indicators.

Firstly, it can be observed from the results that when there exist many nodes in hypernetwork, the ND, NND of some nodes are the same. The importance of these nodes may not be distinguished significantly in the situations of mass nodes or complex collaboration relationships. The $\mathrm{BC}$ and $\mathrm{CC}$ indicators are in similar cases, but our method has relatively high separability.

Secondly, $v_{7}$ is considered to be the most important node by the first four methods. However, as our method integerates direct loss and indirect loss together, despite the direct loss of $v_{7}$ is the largest one, but its indirect loss is less than $v_{13}$, totally $v_{13}$ is calculated as the most important one. In Figure 3 we can see that $v_{7}$ has very close collaborations with $v_{5}, v_{6}, v_{3}$ and $v_{11}$, while $v_{13}$ is connected with nodes in different regions, so when $v_{7}$ is deleted, the influence to the connectiveness of other nodes is limited. On the contrary, if deleting $v_{13}$, the influence to the network connectiveness is relatively greater. That is to say, although a researcher has strong scientific research ability in an organization that collaborates closely, but compared to a researcher collaborates with important researchers in many different organizations, the latter one may have greater influence on the network, which is in line with reality.

In the real network, if there exist more nodes, the network formed is usually more complex and subtle. In the case of the more nodes in hypernetwork, the distinguishing ability of our method will be more significant. It makes our method practical and exact in 
the measuring of big networks, which is very common in many areas in the big data era.

\section{Conclusions}

Hypernetwork has stronger modeling capability than traditional network and has been applied widely recently. In hypernetwork, how to measure the node importance is a critical and fundamental issue which determines the further applications of hypernetwork model. The extant node importance measuring method based on classical theory has limitations towards hypernetwork representations. Based on the idea of deleting methods, this paper utilizes incidence matrix, adjacent matrix, distance matrix of hypernetwork and designs a node importance measuring method. Through being compared with indicators of node degree, neighbor node degree, betweenness centrality and closeness centrality, the effectiveness of our method is demonstrated. The contribution of this work is:

(1) This method takes the whole network and relations between nodes into considerations and avoids the one-sidedness of single indicator, which makes the results being more reliable.

(2) In the case of mass nodes in network, our method can show the discrepancy of nodes importance more significantly, and the accuracy will be higher than single indicators.

(3) The node importance measuring method can lay the foundation for hypernetwork represented problems, such as important researcher discovery in organizations, identification of core host in computer network, and recognition of key group in disease propagations.

Besides the contributions above, there are some topics about this research that can be studied further in future. For example, the calculation model of direct loss and indirect loss, the combination of them and weight setting, and the adaptability for networks with different scales etc.

\section{Acknowledgements}

This work has been supported by National Natural Science Foundation of China (NSFC, Grant No. 71361011 and 61562033), Undergraduate Training Programs for Innovation and Entrepreneurship of JXUFE, Science and Technology Project of Education Department of Jiangxi Province (Grant No. GJJ11734) and JXUFE Research Project. The author would be like to thank the reviewers for their helpful comments and suggestions for improving the manuscript.

\section{References}

[1] S. Slotte-Kock and N. Coviello, "Entrepreneurship Research on Network Processes: A Review and Ways Forward", Entrepreneurship Theory and Practice, vol. 34, no. 1, (2010), pp. 31-57.

[2] J. Liu, G. Yang and Z. Hu, “A Knowledge Generation Model via the Hypernetwork", Plos One, vol. 9, no. 3, (2014), pp. 1-8.

[3] Y. Sheffi, "Urban transportation networks: Equilibrium analysis with mathematical programming methods", Prentice-Hall, Inc. Englewood Cliffs, NJ, (1985).

[4] A. Nagurney, T. Wakolbinger and L. Zhao, "The evolution and emergence of integrated social and financial networks with electronic transactions: a dynamic supernetwork theory for the modeling, analysis, and computation of financial flows and relationship levels", Computational economics, no. 27, (2006), pp. 353-393.

[5] Y. Wu and S. Cai, "Hypergraph Model of prior knowledge in opportunity discovery", International Symposium on Knowledge Acquisition and Modeling, KAM, (2008), pp. 216-220.

[6] P. Bonacich, "Power and centrality: A family of measures", American Journal of sociology, vol. 92, no. 5, (1987), pp. 1170-1182.

[7] H. Jun, B. Wang and D. Lee, "Evaluating Node Importance with Multi-criteria", Proceeding of IEEE/ACM International Conference on Green Computing and Communications and IEEE/ACM International Conference on Cyber, Physical and Social Computing, (2010), pp. 792-797.

[8] D. Schall, "Measuring contextual partner importance in scientific collaboration networks", Journal of Informetrics, vol. 7, no. 3, (2013), pp. 730-736. 
[9] T. Agryzkov, J. L. Oliver and L. Tortosa, "A new betweenness centrality measure based on an algorithm for ranking the nodes of a network", Applied Mathematics and Computation, vol. 244, (2014), pp. 467-478.

[10] P. Li, Y. Ren and Y. Xi, “An importance measure of actors (set) within a network", Syst. Eng., vol. 22, no. 4, (2004), pp. 13-20.

[11] X. Qi, E. Fuller and R. Luo, "A novel centrality method for weighted networks based on the Kirchhoff polynomial”, Pattern Recognition Letters, vol. 58, (2015), pp. 51-60.

[12] K. Saito, M. Kimura and K. Ohara, "Super mediator - A new centrality measure of node importance for information diffusion over social network", Information Sciences, vol. 329, (2016), pp. 985-1000.

[13] A. Landherr, B. Friedl and J. Heidemann, "A Critical Review of Centrality Measures in Scocial Networks", Business \& Information Systems Engineering, vol. 2, no. 6, (2010), pp. 371-385.

[14] C. Berge, "Graph and hypergraph", Amsterdam: North Holland, (1973).

[15] Q. Xiao, "Scientific Research Collaboration Hypernetwork Modeling and Node Importance Measuring", 2014 International Conference on Management of e-Commerce and e-Government, ICMeCG, (2014), pp. 110-115.

[16] E. Estrada and J. A. Rodriguez-Velazquez, "Subgraph Centrality in Complex Networks", Physical Review E, vol. 71, no. 5, (2005), pp. 1-9.

[17] U. Brandes, "A fast algorithm for betweenness centrality", Journal of Mathematical Sociology, vol. 25, no. 2, (2001), pp. 163-177.

[18] M. E. J. Newman, "A measure of betweenness centrality based on random walk", Social Networks, vol. 27, no. 1, (2005), pp. 39-54.

[19] K. Goh, E. Kahng and D. Kim, "Betweenness centrality correlation in social networks", Phys Rev.E, vol. 67, no. 1, (2003), 017101.

\section{Authors}

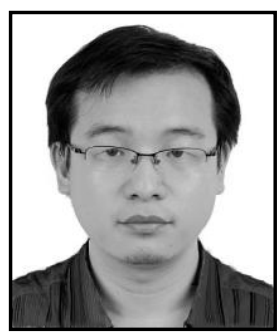

Quan Xiao, he received his Ph.D. degree in management science and engineering from Huazhong University of Science and Technology, China in 2010. He is currently a lecturer in the School of Information Technology, Jiangxi University of Finance and Economics, China. His research interests include decision support systems, risk identification and online customer behaviors. 